\title{
Hydrogel-Based Ultra-moisturizing Cream Formulation for Skin Hydration and Enhanced Dermal Drug Delivery
}

\author{
Sang Gon Lee, ${ }^{a}$ Sung Rae Kim, ${ }^{a}$ Hye In Cho, ${ }^{a}$ Mean Hyung Kang, ${ }^{a}$ Dong Woo Yeom, ${ }^{a}$ \\ Seo Hyun Lee, ${ }^{a}$ Sangkil Lee, ${ }^{b}$ and Young Wook Choi*,a \\ ${ }^{a}$ College of Pharmacy, Chung-Ang University; 221 Heuksuk-dong, Dongjak-gu, Seoul 156-756, Republic of \\ Korea: and ${ }^{b}$ College of Pharmacy, Keimyung University; 1095 Dalgubeol-daero, Daegu 704-701, Republic of Korea. \\ Received June 19, 2014; accepted July 19, 2014
}

To develop an external vehicle for skin hydration and enhanced dermal drug delivery, a hydrogel-based ultra-moisturizing cream (HUMC) was successfully formulated with carbopol 934P, urea, Tinocare GL, grape seed oil, and other excipients. The HUMC showed plastic flow behavior due to a gel structure with a cream base. Different types of drug-free vehicles such as a hydrogel, conventional cream (CC), and three HUMCs were prepared and subjected to an in vivo skin hydration test on a hairless mouse using a corneometer. Hydration effect $(\triangle \mathrm{AU})$ was in the order of HUMC2 $>$ HUMC1 $\geq$ CC $>$ HUMC3 $>$ hydrogel. Using nile red (NR) and 5-carboxyfluorescein (5-CF) as lipophilic and hydrophilic fluorescent probes, respectively, in vitro skin permeation and accumulation studies were conducted using Franz diffusion cells. The values of steady-state flux $\left(J_{\mathrm{ss}}, \mathrm{ng} / \mathrm{h} / \mathrm{cm}^{2}\right)$ were obtained: 74.8 (CC), 145.6 (HUMC1), and 161.9 (HUMC2) for NR delivery; 6.8 (CC), 8.3 (HUMC1), and 10.9 (HUMC2) for 5-CF delivery. The amounts retained in the skin at $12 \mathrm{~h}$ $\left(Q_{\mathrm{r}}, \mathrm{ng} / \mathrm{cm}^{2}\right)$ were determined: 86.4 (CC) and 102.0 (HUMC2) for NR; and 70.1 (CC) and 195.6 (HUMC2) for 5-CF. Confocal microscopy was used to visualize the distribution of the fluorescent probes. NR tended to be localized into the deeper part of the skin with adipose tissue whereas 5-CF localized in the upper layer of the skin. Thus we propose that HUMC2 is an efficacious vehicle for skin hydration and enhances dermal delivery of lipophilic and hydrophilic drugs. bution

Key words hydrogel-based cream; moisturizer; skin hydration; topical delivery; skin permeation; skin distri-

Skin dryness is induced by complex interactions between environmental and individual factors and is frequently observed in skin diseases such as atopic dermatitis (AD), psoriasis, and ichthyosis. ${ }^{1,2)} \mathrm{AD}$ is a chronic inflammatory skin disease, frequently occurring in infancy and childhood. The stratum corneum (SC) of AD patients is less hydrated and less capable of binding water than normal skin. Dryness of the skin can induce pruritus in $\mathrm{AD}$, aggravating the skin disease. ${ }^{3)}$ A vicious itch-scratch cycle is established, because scratching facilitates the release of pro-inflammatory mediators that worsen itching. ${ }^{4)}$ The use of moisturizers is recommended for treatment of the above skin diseases. ${ }^{5)}$ Moisturizers have a steroid-sparing effect in inflammatory skin disorders such as AD. ${ }^{6)}$ After application of moisturizers, the water content in the SC increases and smoothing of the surface can be observed as a result of filling of the spaces between partially desquamated skin flakes. ${ }^{7)}$ There are at least two different means of SC hydration: occlusion of the skin surface and introduction of humectants. Occlusion with lipids such as fats and oil forms a greasy film, reducing water loss from the SC. ${ }^{8)}$ Humectants such as urea and glycerin are absorbed into the SC, amplifying skin hydration by attraction of water from dermis into the $\mathrm{SC}^{9}{ }^{9}$ )

The SC, the outermost layer of the skin, comprises 12-16 layers of corneocytes, and when dry, it has a thickness of $10-15 \mu \mathrm{m}$. The structure of the SC is often depicted in the so-called brick and mortar arrangement, where the keratinrich corneocytes (bricks) are embedded in the intercellular lipid-rich matrix (mortar). ${ }^{10)}$ Permeability of drugs in the skin is very limited because the $\mathrm{SC}$ acts as the main skin barrier.

The authors declare no conflict of interest.
Numerous studies have described the potential use of chemical enhancers such as alcohols, dimethyl sulfoxide, and azone analogues to promote skin penetration by reducing the barrier capability of the SC. ${ }^{11,12)}$ However, these chemical enhancers can trigger skin irritation. As an alternative, skin moisturization is recommended to increase the skin penetration of drugs, since moisturization may lead to swelling and opening of the SC structure. ${ }^{13)}$

Creams and hydrogels are the most commonly used topical preparations for skin moisturization. Creams are two-phase systems containing oil and water, one of which is dispersed in the other in the form of microscopic droplets. In order to achieve a homogeneous dispersion of the droplets, emulsifiers are added to the cream. Some of these emulsifiers can cause irritation on dry skin. ${ }^{14)}$ In contrast, hydrogels, which do not contain emulsifiers, may reduce skin irritation and adequately hydrate dry skin.

Carbomer (carbopol 934P), an acrylic acid polymer crosslinked with polyalkenyl polyethers or divinyl glycol, is one of the most commonly used hydrophilic polymers for gel formula. Hydrogels have some advantages such as high viscosity at low concentrations, compatibility with many active ingredients, bioadhesive properties, thermal stability, and patient acceptance. ${ }^{15)}$ However, hydrogels can leave a dry scale-like film after application, because of solvent evaporation. ${ }^{16)}$ Ideally, a combination of a hydrogel and a cream could offer another option for effective treatment of dry skin diseases. The addition of a polymeric base in a cream results in the formation of a hydrogel-based cream with a networked gel structure in the water phase. A hydrogel-based cream with an oil component forms a greasy polymeric film on the skin and improves skin hydration by preventing transepidermal water loss, thereby 
potentially enhancing the dermal delivery of drug molecules.

The aim of the present study was to develop a hydrogelbased ultra-moisturizing cream (HUMC) for enhanced skin hydration and topical drug delivery. The effect of formulation factors including the type and/or concentration of ingredients on skin hydration was evaluated. Using nile red (NR) and 5-carboxyfluorescein (5-CF) as hydrophobic and hydrophilic fluorescent probes, respectively, in vitro skin permeation and accumulation were quantitatively measured. Skin distribution of the fluorescent probes was visualized using confocal microscopy.

\section{MATERIALS AND METHODS}

Animals Male, five-week-old, hairless mice (SKH:HR-1) were purchased from Orient Bio (Kyunggi, Korea). They were kept in specific pathogen-free conditions with food and water freely available. All animal experiments were performed in accordance with the NIH "Principles of Laboratory Animal Care" guidelines (NIH Publication No. 85-23, revised 1996) and were approved by the Institutional Animal Care and Use Committee of Chung-Ang University, Seoul, Korea.

Materials Carbopol 934P and Tinocare GL (scleroglucan polysaccharide) were kindly provided by Masung Co., Ltd. (Seoul, Korea) and BASF (Ludwigshafen, Germany), respectively. Tegocare 450 (polyglyceryl-3-methyl glucose distearate), grape seed oil, mineral oil, stearic acid, and cetyl alcohol were generously provided by Interlees Ltd. (Seongnam, Korea). Olivem 1000 (sorbitan ester and cetearyl ester of olive oil fatty acids) was provided by B\&T (Milano, Italy). Carnosine was purchased from Tokyo Kasei Kogyo Co., Ltd. (Tokyo, Japan). NR, 5-CF, glycerin, and DL- $\alpha$-tocopherol acetate were purchased from Sigma-Aldrich Co. (St. Louis, MO, U.S.A.). Urea was purchased from Daejung Chemicals (Si-heung, Korea). All other chemicals and reagents obtained from commercial sources were of analytical grade. Double distilled water was used for all experiments.

Preparation of Test Formulations Different types of drug-free vehicles such as hydrogel, conventional cream (CC), and HUMCs were prepared and tested in an in vivo skin hydration test. As shown in Table 1, a polymeric hydrogel base was prepared with carbopol 934P $(0.5 \%)$ dispersed in deionized water and glycerin (5\%), followed by neutralization with carnosine $(0.5 \%)$. HUMC vehicles were prepared by homogenization (Ultra-Turrax T25; $13000 \mathrm{rpm}, 10 \mathrm{~min}$ ) of a melted oil phase (lipids, emollients, and emulsifier) with an aqueous phase (hydrogel base and humectants). Urea and Tinocare GL were added as humectants. The oil phase consisted of grape seed oil as an emollient. Olivem 1000 was used as a non-irritant emulsifier, and DL- $\alpha$-tocopherol acetate as an antioxidant. Stearic acid and cetyl alcohol were selectively added to the oil phase to increase occlusiveness. The dispersions obtained were neutralized with carnosine $(0.5 \%)$. The product was degassed under vacuum and cooled to ambient temperature. Separately, CC was prepared by homogenization of oil phase (mineral oil, stearic acid, Tegocare 450, and cetyl alcohol) with the aqueous phase (glycerin and water) under the conditions described above. Fluorescent probe-loaded CC and HUMC formulations were prepared for in vitro skin permeation and accumulation studies by the addition of NR $(0.005$ $\mathrm{w} / \mathrm{w} \%)$ and $5-\mathrm{CF}(0.02 \mathrm{w} / \mathrm{w} \%)$ in oil and aqueous phases, respectively, prior to homogenization.

Properties of HUMCs Organoleptic (color, thickness, appearance, and texture) and physical (creaming and phase separation) properties of the HUMCs were assessed. Upon storage of the samples at temperatures of 25 and $40^{\circ} \mathrm{C}, \mathrm{pH}$ values were measured using a digital $\mathrm{pH}$ meter (EcoMet P25, iSTEK, Korea) and the centrifugal separation test was performed at $3000 \times \boldsymbol{g}$ for $10 \mathrm{~min}$ by using a Micro $17 \mathrm{TR}$ centrifuge (Hanil Scientific Industrial, Korea). To determine the type of HUMC, dilution tests were performed: oil-in-water $(\mathrm{O} / \mathrm{W})$ or water-inoil (W/O) type was determined if the HUMC homogeneously dispersed with water or light mineral oil, respectively. ${ }^{17)}$

Rheological Evaluation Viscosity was assessed using a Bohlin CVO rheometer (Malvern Instruments, Malvern, U.K.) with a CP 4/40 cone and plate fixture ( $4^{\circ}$ cone angle, $40-\mathrm{mm}$ cone diameter). The distance between the cone and plate was set to $150 \mu \mathrm{m}$ for all measurements. Experiments were conducted under a steady shear flow at $25^{\circ} \mathrm{C}$. Shear rates ranged from 0.01 to $300 \mathrm{~s}^{-1}$. Hydrogel, CC, and HUMC vehicles were

Table 1. Composition of Various Vehicles

\begin{tabular}{|c|c|c|c|c|c|}
\hline Ingredients & $\mathrm{CC}$ & Hydrogel & HUMC1 & HUMC2 & HUMC3 \\
\hline \multicolumn{6}{|l|}{ Polymeric base } \\
\hline Carbopol 934P & - & 0.5 & 0.5 & 0.5 & 0.5 \\
\hline Carnosine & - & 0.5 & 0.5 & 0.5 & 0.5 \\
\hline \multicolumn{6}{|l|}{ Oil phase } \\
\hline Cetyl alcohol & 2 & - & - & 1 & 2 \\
\hline Grape seed oil & - & - & 4 & 4 & 4 \\
\hline Mineral oil & 7 & & & & \\
\hline Olivem 1000 & - & - & 0.5 & 0.5 & 0.5 \\
\hline Stearic acid & 5 & - & - & 2 & 3 \\
\hline Tegocare 450 & 3 & & & & \\
\hline DL- $\alpha$-Tocopherol acetate & - & - & 0.5 & 0.5 & 0.5 \\
\hline \multicolumn{6}{|l|}{ Aqueous phase } \\
\hline Urea & - & - & 3 & 3 & 3 \\
\hline Tinocare GL & - & - & 10 & 10 & 10 \\
\hline Glycerin & 10 & 5 & 5 & 5 & 5 \\
\hline Distilled water & & & q.s. ad 100.0 & & \\
\hline
\end{tabular}

CC, conventional cream; HUMC, hydrogel-based ultra-moisturizing creams. 
tested. All experiments were performed in triplicate and averaged.

In Vivo Skin Hydration Studies Corneometer CM 820 (Courage+Khazaka Company, Cologne, Germany) was used to evaluate skin hydration by measuring capacitance, expressed as an arbitrary unit (A.U.). The degree of skin hydration was defined as the changes in A.U. values $(\Delta \mathrm{AU})$ measured before and after application of test formulations. The measurements were performed under ambient conditions with constant relative humidity $(45 \pm 5 \%)$ and a constant temperature $\left(22 \pm 3^{\circ} \mathrm{C}\right)$. Prior to all measurements, mice were kept under experimental conditions for at least $1 \mathrm{~h}$ in order to allow skin adaptation. The following formulations were tested: CC, hydrogel, HUMCs, and commercial products (ATOPALM $^{\circledR}$, Neutrogena moisturizing cream $^{\circledR}$, and Baviola RF cream $^{\circledR}$ ). After application of each formulation $(100 \mu \mathrm{L})$ to the central dorsal skin of the mice $\left(1 \mathrm{~cm}^{2}\right)$, measurements were performed at predetermined time points.

In Vitro Skin Permeation Studies In vitro permeation studies were conducted using the vertical-type Franz diffusion cell. Skin tissues were obtained from hairless mice. A circular piece of dorsal skin was carefully mounted onto the diffusion cell with the SC facing in the direction of the donor compartment, which had an available diffusion area of $1.76 \mathrm{~cm}^{2}$. To maintain sink conditions, the receptor chamber was filled with either $50 \%$ ethanolic solution for NR or $10 \mathrm{~mm}$ PBS $(\mathrm{pH}$ 7.4) solution for $5-\mathrm{CF}$, and was maintained at $37^{\circ} \mathrm{C}$ by using a water jacket. Test formulations containing equivalent amount of NR $(25 \mu \mathrm{g})$ or $5-\mathrm{CF}(100 \mu \mathrm{g})$ were applied to the skin surface. All experiments were carried out with an occluded donor compartment. Aliquots $(0.5 \mathrm{~mL})$ were withdrawn from the receptor compartment at predetermined time points and analyzed using HPLC. The cumulative amount of drug permeated per unit area was plotted as a function of time, and the steadystate permeation rate $\left(J_{\mathrm{ss}}\right)$ was calculated from the slope.

In Vitro Skin Accumulation Studies The amount of $\mathrm{NR}$ or $5-\mathrm{CF}$ retained in the skin was determined after $12 \mathrm{~h}$ in the in vitro skin permeation studies. The barrier skin was washed several times with distilled water and then gently dried by pressing between two tissues. A sample of mouse skin $\left(1.76 \mathrm{~cm}^{2}\right)$ was cut into small pieces using scissors. The tissue was further homogenized with methanol solution $(50 \%)$ for $5 \mathrm{~min}$ and sonicated in a bath sonicator (Model 2210, Branson Ultrasonics Co., U.S.A.) for $30 \mathrm{~min}$ at $25^{\circ} \mathrm{C}$. The resulting solution was centrifuged for $10 \mathrm{~min}$ at $5000 \mathrm{rpm}$. The NR or 5-CF content in the supernatant was determined using HPLC.

Confocal Microscopic Examination for Skin Distribution To visualize the distribution of $\mathrm{NR}$ and 5-CF in the skin over time, the skin sample was removed from the Franz diffusion cell at $3 \mathrm{~h}$ and $6 \mathrm{~h}$. After washing and drying, the skin was cut with a scalpel to the proper size. The skin specimen was positioned vertically in the bottom of a mold and surrounded with Tissue-Tek ${ }^{\circledR}$ O.C.T compound 4583 (Sakura Finetek, U.S.A.), and rapidly frozen under liquid nitrogen. The frozen skin was sliced vertically at a thickness of $30 \mu \mathrm{m}$ by using Cryotome (Ultracut UCT, Leica Microsystems, Germany) at $-25^{\circ} \mathrm{C}$. The skin slices were observed using confocal laser scanning microscopy (CLSM 510, Carl Zeiss, Germany). NR was excited at $543 \mathrm{~nm}$ and its emission was detected using a long-pass filter (LP 560, Carl Zeiss). 5-CF was excited at $488 \mathrm{~nm}$ and its emission was detected using a long-pass filter (LP 505, Carl Zeiss).

HPLC Assay for NR and 5-CF The quantitative determination of NR and 5-CF was performed using a HPLC system consisting of a pump (L-7100), fluorescence detector (L-7485), a data station (L-7000, Hitachi, Japan), and a C18 column $(0.5 \mu \mathrm{m}, 4.6 \times 150 \mathrm{~mm}$; Shiseido, Tokyo, Japan). For the NR assay, methanol-water $(93: 7, \mathrm{v} / \mathrm{v})$ was used as the mobile phase at a flow rate of $1 \mathrm{~mL} / \mathrm{min}$. The system was set at 559 and $630 \mathrm{~nm}$ for excitation and emission wavelengths, respectively. The NR peak was separated with a retention time of $3.5 \mathrm{~min}$. For the 5-CF assay, the mobile phase for isocratic elution was a mixture of $10 \mathrm{~mm}$ sodium phosphate $(\mathrm{pH} 7.05)$ and methanol $(8: 2)$ at a flow rate of $1 \mathrm{~mL} / \mathrm{min}$. The excitation and emission wavelengths were set at 492 and $517 \mathrm{~nm}$, respectively. The 5-CF peak was separated with a retention time of $4 \mathrm{~min}$.

Statistical Analysis All reported data are represented as mean \pm S.D. $(n=3)$. Statistical significance was determined using Student's $t$-test and set at $p<0.05$, unless otherwise indicated.

\section{RESULTS}

Characterization of HUMC Formulation The characteristics of the HUMC vehicle are summarized in Table 2. The vehicle had cream-like properties with a gleaming white tone. It was homogenous, non-greasy, and smooth in texture. On application, it did not leave any greasiness. The results from dilution test indicated that the HUMC vehicle belonged to an $\mathrm{O} / \mathrm{W}$ system and there was no phase separation after centrifugation in any of the samples until $28 \mathrm{~d}$. The $\mathrm{pH}$ values for $\mathrm{CC}$ and HUMC vehicles stored at 25 or $40^{\circ} \mathrm{C}$ were maintained in the range of 4.66 to 5.67 for $28 \mathrm{~d}$ (Table 3 ).

The rheological properties of hydrogel and HUMC vehicles were shown in Fig. 1. HUMC was a non-Newtonian liquid and belonged to a shear-thinning system, because the viscosity was initially high, but decreased with increasing shear rate. With regard to rheological behavior, HUMC was categorized as a plastic fluid, identified based on the appearance of a yield stress, i.e., the fluid flows in a viscous manner only after a threshold has been exceeded. Below this threshold, known as the yield stress, the behavior of the fluid is similar to that of an elastic solid.

Table 2. Characteristics of Hydrogel-Based Ultra-moisturizing Creams (HUMCs)

\begin{tabular}{ll}
\hline \hline Characterization & \multicolumn{1}{c}{ Results } \\
\hline Organoleptic property & Homogenous, non-greasy, smooth texture, white \\
Type of interposition & Oil-in-water $(\mathrm{O} / \mathrm{W})$ system \\
Centrifugal test & No separation until 28d \\
Rheological evaluation & Plastic flow behavior \\
\hline
\end{tabular}


Table 3. $\mathrm{pH}$ Values of Conventional Cream (CC) and Hydrogel-Based Ultra-moisturizing Creams (HUMCs) Stored at $25^{\circ} \mathrm{C}$ and $40^{\circ} \mathrm{C}$

\begin{tabular}{|c|c|c|c|c|c|c|c|c|}
\hline \multirow{2}{*}{ Time } & \multicolumn{4}{|c|}{$25^{\circ} \mathrm{C}$} & \multicolumn{4}{|c|}{$40^{\circ} \mathrm{C}$} \\
\hline & $\mathrm{CC}$ & HUMC1 & HUMC2 & HUMC3 & $\mathrm{CC}$ & HUMC1 & HUMC2 & HUMC3 \\
\hline $0 \mathrm{~h}$ & 5.01 & 5.35 & 5.14 & 5.02 & - & - & - & - \\
\hline $12 \mathrm{~h}$ & 5.24 & 5.24 & 5.17 & 5.03 & 4.76 & 5.37 & 5.07 & 5.06 \\
\hline $24 \mathrm{~h}$ & 4.82 & 5.55 & 5.27 & 5.12 & 4.74 & 5.32 & 5.29 & 5.03 \\
\hline $36 \mathrm{~h}$ & 4.96 & 5.52 & 5.25 & 5.19 & 4.75 & 5.41 & 5.19 & 5.13 \\
\hline $48 \mathrm{~h}$ & 5.20 & 5.55 & 5.26 & 5.24 & 5.06 & 5.46 & 5.41 & 5.23 \\
\hline $72 \mathrm{~h}$ & 4.94 & 5.67 & 5.34 & 5.28 & 4.93 & 5.38 & 5.32 & 5.35 \\
\hline $7 d$ & 4.66 & 5.60 & 5.19 & 5.13 & 4.74 & 5.33 & 5.20 & 5.12 \\
\hline $14 \mathrm{~d}$ & 5.21 & 5.57 & 5.33 & 5.28 & 4.81 & 5.57 & 5.56 & 5.40 \\
\hline $21 \mathrm{~d}$ & 5.36 & 5.17 & 5.22 & 5.15 & 5.03 & 5.51 & 5.67 & 5.49 \\
\hline $28 \mathrm{~d}$ & 5.36 & 5.11 & 5.28 & 5.12 & 5.01 & 5.53 & 5.60 & 5.38 \\
\hline
\end{tabular}
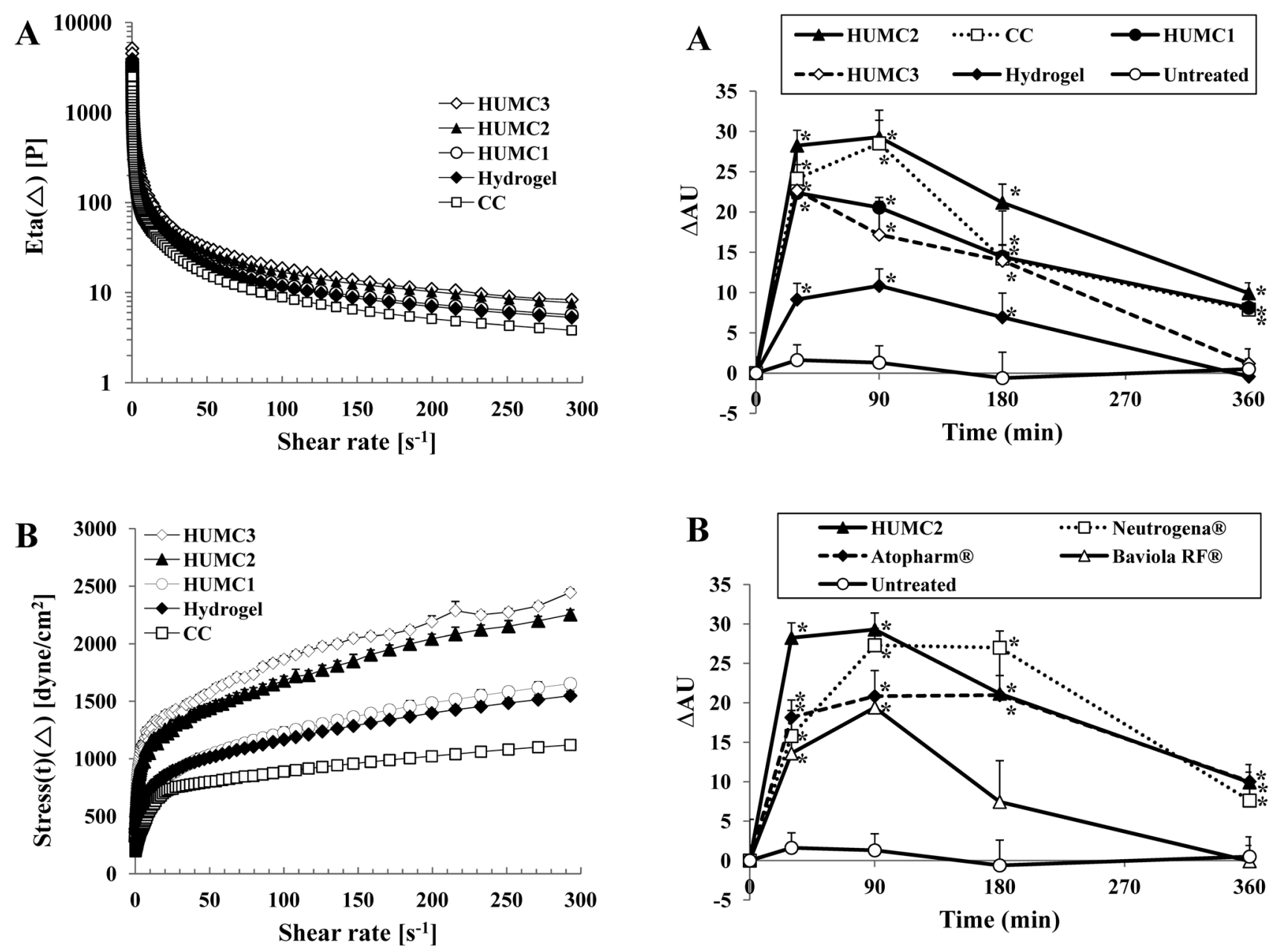

Fig. 1. Viscosity Curve (A) and Flow Curve (B) of Hydrogel, Conventional Cream (CC), and Hydrogel-Based Ultra-moisturizing Creams (HUMCs)

Data represent mean \pm S.D. $(n=3)$.

In Vivo Skin Hydration The skin hydration effects of $\mathrm{CC}$ and HUMC vehicles in hairless mice are shown in Fig. 2A. The untreated group showed negligible skin hydration with a constant corneometer value. In contrast, for all treated groups, skin hydration increased rapidly within $30 \mathrm{~min}$ after application, lasted for about $60 \mathrm{~min}$, and then decreased gradually. Although this pattern was similar in all test formulations, the degree of hydration was quite different. The hydrogel was

Fig. 2. Changes in Skin Hydration Values in Hairless Mice after Topical Administration of Various Formulations: (A) Hydrogel, Conventional Cream (CC), and Hydrogel-Based Ultra-moisturizing Creams (HUMCs); (B) HUMC2 and Commercial Formulations

Data represent mean \pm S.D. $(n=5)$. $*$ Statistical differences $(p<0.05)$ between treated groups with various formulations and untreated group (control).

less effective compared to both HUMC and CC formulations. HUMC2 was superior to other formulations, resulting in the greatest $\triangle \mathrm{AU}$ at all time points observed. At the time of termination (6h), the order of $\Delta \mathrm{AU}$ values was as follows: HUMC2 (9.91) $>$ HUMC1 $\quad(8.19) \geq \mathrm{CC} \quad(7.89)>$ HUMC3 $\quad(1.18)>$ hydrogel (0.3). In Fig. 2B, the best formulation in the prior hydration study (HUMC2) was compared with other commercially 

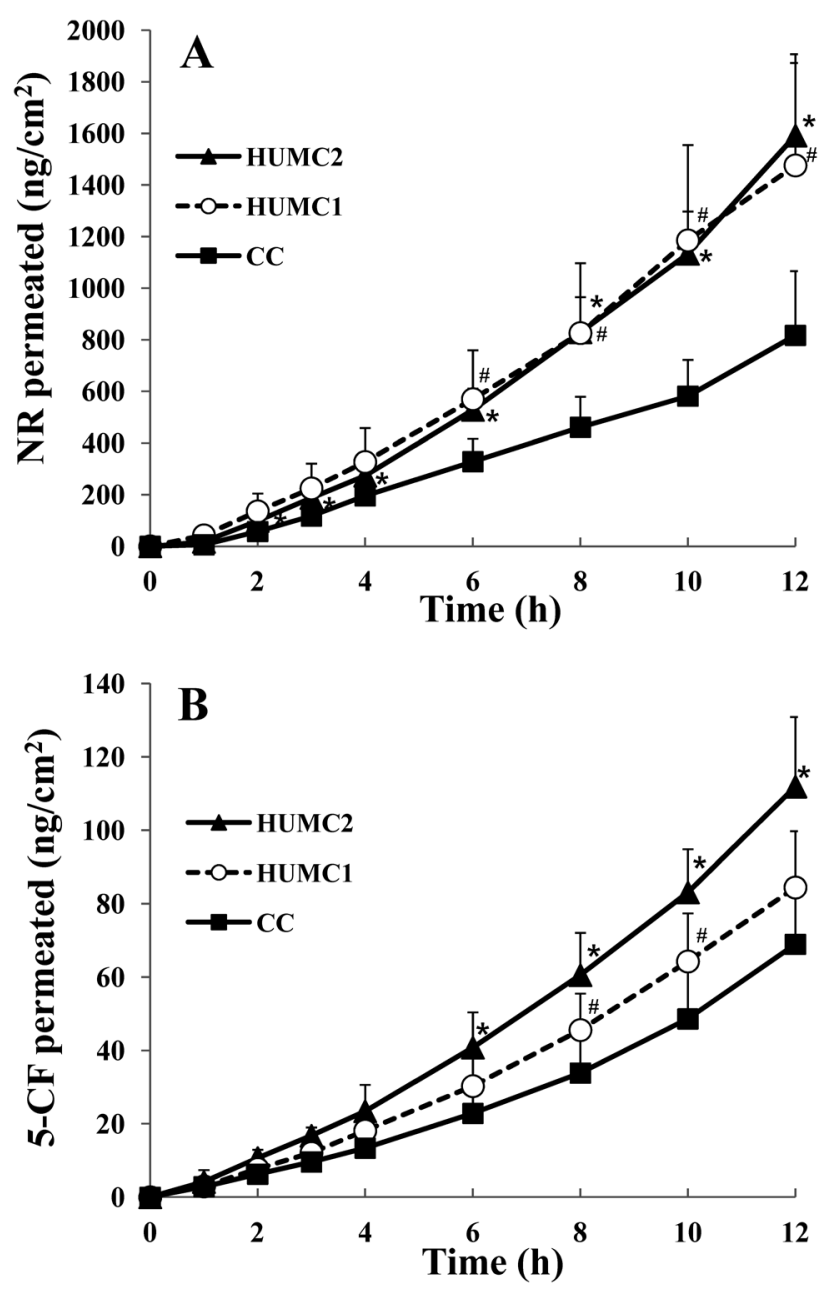

Fig. 3. Permeation Profiles of Nile Red (NR) (A) and 5-Carboxyfluorescein (5-CF) (B) in Conventional Cream (CC) and Hydrogel-Based Ultramoisturizing Creams (HUMCs) through Hairless Mouse Skin

Data represent mean \pm S.D. $(n=6)$. * Statistical difference $(p<0.05)$ between HUMC2 and CC; " Statistical difference $(p<0.05)$ between HUMC1 and CC.

available moisturizing products. HUMC2 showed a sharp increase in $\Delta \mathrm{AU}$ at $30 \mathrm{~min}$, but other products showed a gradual increase until $90 \mathrm{~min}$. The maximum values for $\Delta \mathrm{AU}$ were HUMC2 (29.29)>Neutrogena (27.30)>Atopharm (20.98)>Baviola RF (19.41).

In Vitro Skin Permeation We evaluated the skin permeation profiles of $\mathrm{NR}$ and 5-CF in $\mathrm{CC}$ and $\mathrm{HUMC}$ formulations. The cumulative amount of $\mathrm{NR}$ and $5-\mathrm{CF}\left(\mathrm{ng} / \mathrm{cm}^{2}\right)$ in receptor phase was plotted as a function of time (Fig. 3). NR and 5-CF permeated the skin barrier with no distinct lag time in all formulations, indicating that steady-state of permeation was reached rapidly. As shown in Fig. 3a, during the initial period of $3 \mathrm{~h}$, permeation profiles of NR were similar for both $\mathrm{CC}$ and HUMC formulations. Afterward, however, the slope of the HUMC formulations was much steeper than that of CC. In a separate experiment, permeation of 5-CF from $\mathrm{CC}$ and HUMC formulations was compared (Fig. 3b). For the initial $3 \mathrm{~h}$ period, the flux was similar for the different formulations. However, after $3 \mathrm{~h}$, the flux of $5-\mathrm{CF}$ was revealed as HUMC2 $>$ HUMC1 $>$ CC.

The parameters for skin permeation of $\mathrm{NR}$ and 5-CF from different formulations are summarized in Table 4. The flux $\left(J_{\mathrm{ss}}\right)$ values of NR permeation were significantly higher than those of 5-CF permeation, showing an order of magnitude difference. The amount permeated (percentage) was calculated by dividing the cumulative amount in the receptor phase at $12 \mathrm{~h}$ by the total amount in the sample loaded to the donor compartment; NR was in the range of 5 to $10 \%$, but 5 -CF was less than $0.2 \%$. As expected, regardless of formulation type, the lipophilic probe more easily permeated the skin barrier than the hydrophilic probe. Nevertheless, HUMC formulations effectively increased the permeation of both probes, suggesting their superiority over the $\mathrm{CC}$ formulation. For further comparison, enhancement ratio (ER) was expressed as a relative ratio of the flux values. Both HUMC1 and HUMC2 formulations produced a large increment (about 2-fold) in NR delivery. In the case of 5-CF delivery, HUMC1 and HUMC2 produced 1.2- and 1.6-fold increments, respectively.

In Vitro Skin Accumulation At the termination of skin permeation studies at $12 \mathrm{~h}$, the skin was collected and assayed for the amount of NR or 5-CF retained $\left(Q_{\mathrm{r}}, \mathrm{ng} / \mathrm{cm}^{2}\right)$. As listed in Table 4 , the average $Q_{\mathrm{r}}$ values for NR were in the range of 86 to 112 based on the formulation type. Even though HUMC formulations increased the $Q_{\mathrm{r}}$ values compared to CC, the differences were insignificant. In contrast for 5-CF, average $Q_{\mathrm{r}}$ values were increased by HUMC formulations to three times the $\mathrm{CC}$ level, revealing significant differences between $\mathrm{CC}$ and HUMCs. If we focus on the lipophilicity and/or hydrophilicity of the model fluorescent probes, CC brought a similar level of skin accumulation for both molecules, but HUMCs promoted the skin accumulation of hydrophilic probe to a greater extent. This trend is inconsistent with the results obtained in the skin permeation studies: i.e., parallel or synergistic effect between skin permeation and skin accumulation were not observed.

Visualization of Skin Distribution Figure 4 shows confocal microscopic images of vertical skin sections following the application of CC and HUMC formulations. At $3 \mathrm{~h}$ after the application, the fluorescence appeared to have spread out

Table 4. Skin Permeation Parameters of Nile Red (NR) and 5-Carboxyfluorescein (5-CF) in Conventional Cream (CC) and Hydrogel-Based Ultramoisturizing Creams (HUMCs) through Hairless Mouse Skin

\begin{tabular}{|c|c|c|c|c|c|c|}
\hline & \multicolumn{3}{|c|}{ Nile red } & \multicolumn{3}{|c|}{ 5-Carboxyfluorescein } \\
\hline & $\mathrm{CC}$ & HUMC1 & HUMC2 & $\mathrm{CC}$ & HUMC1 & HUMC2 \\
\hline$J_{\mathrm{ss}}\left(\mathrm{ng} / \mathrm{h} / \mathrm{cm}^{2}\right)^{a)}$ & $74.80 \pm 23.43$ & $145.66 \pm 49.27$ & $161.98 \pm 27.35$ & $6.85 \pm 0.73$ & $8.33 \pm 1.27$ & $10.96 \pm 1.85$ \\
\hline Permeated $(\%)^{b)}$ & $5.75 \pm 1.75$ & $10.39 \pm 3.04$ & $11.20 \pm 1.98$ & $0.12 \pm 0.02$ & $0.15 \pm 0.03$ & $0.20 \pm 0.03$ \\
\hline$Q_{\mathrm{r}}\left(\mathrm{ng} / \mathrm{cm}^{2}\right)^{c)}$ & $86.46 \pm 24.19$ & $112.15 \pm 23.91$ & $102.08 \pm 19.23$ & $70.14 \pm 30.42$ & $210.73 \pm 45.85$ & $195.60 \pm 41.92$ \\
\hline $\mathrm{ER}^{d)}$ & - & 1.95 & 2.17 & - & 1.22 & 1.60 \\
\hline
\end{tabular}

Data represent mean \pm S.D. $(n=6) . a)$ Steady-state flux obtained from the slope of the permeation profile in Fig. 3. $b$ ) Calculated by the ratio of the cumulative amount in the receptor phase at $12 \mathrm{~h}$ versus the total amount in the sample loaded to the donor compartment. $c$ ) The amount retained in the skin at $12 \mathrm{~h}$. $d$ ) Enhancement ratio of steady-state flux, relative to $\mathrm{CC}$. 

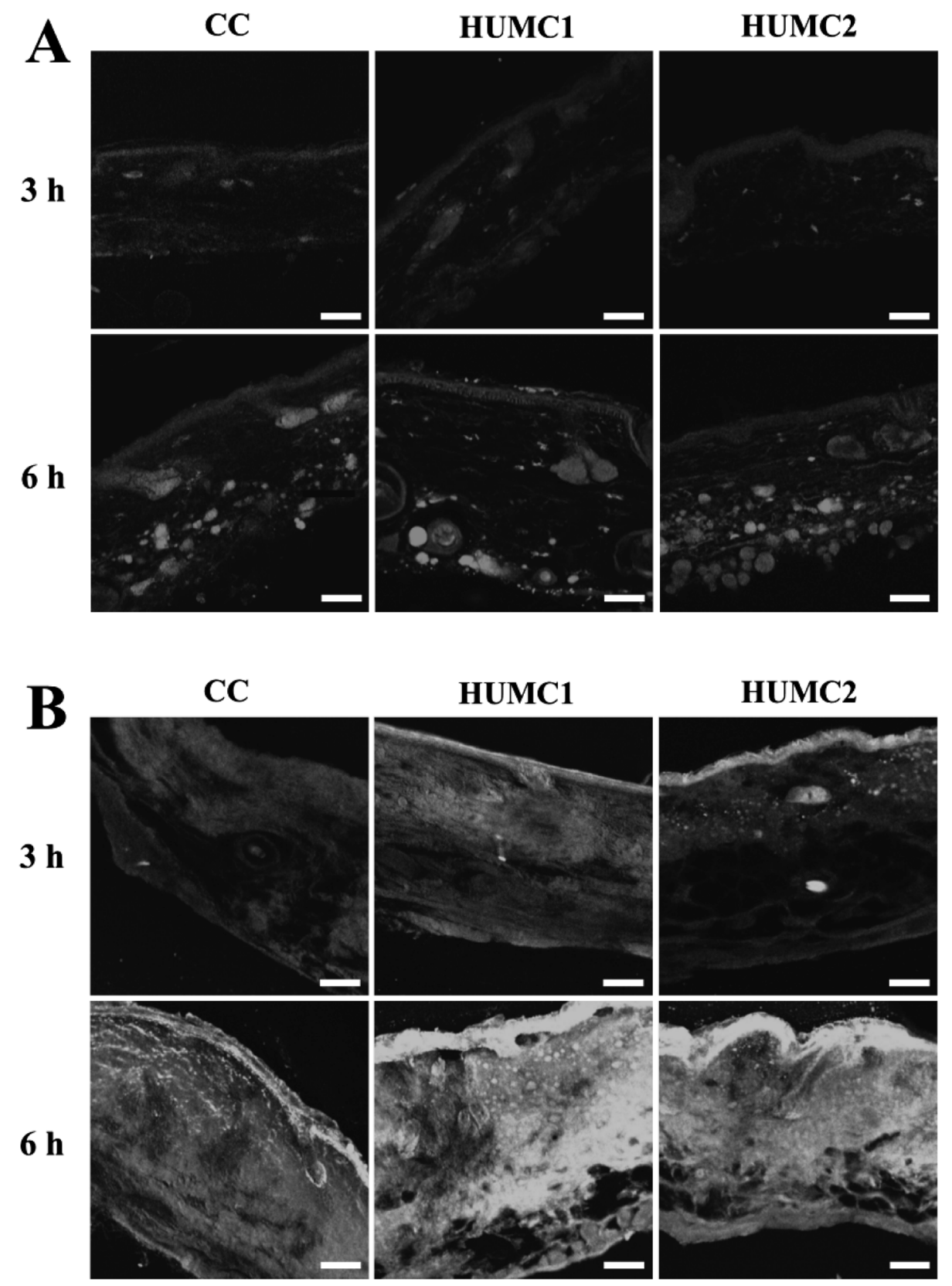

\section{$6 \mathrm{~h}$}

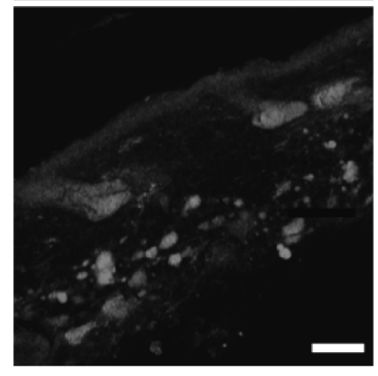

HUMC1

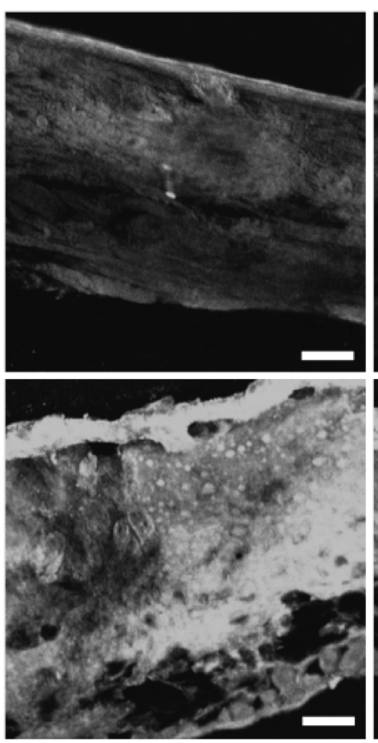

HUMC2

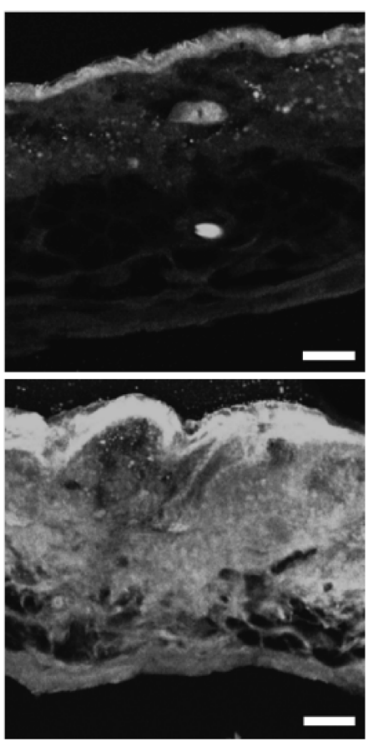

Fig. 4. Confocal Laser Scanning Microscopy Images Revealing Skin Penetration and Distribution of Nile Red (NR) (A) and 5-Carboxyfluoroscein (5-CF) (B) in Conventional Cream (CC) and Hydrogel-Based Ultra-moisturizing Creams (HUMCs)

The skin was vertically cross-sectioned using a cryotome. The white bar represents $100 \mu \mathrm{m}$.

radially. At $6 \mathrm{~h}$, the fluorescence was found in deeper skin layers, scattered throughout the dermal tissues. The fluorescence intensity increased as time elapsed. NR tended to be localized in the deeper part of the skin where adipose tissue was found, but 5-CF was localized to the upper layer of the skin. In observing distribution of the NR probe, formulation effects between $\mathrm{CC}$ and HUMCs were rarely observed (Fig. 4a). However, in case of 5-CF, HUMCs revealed much stronger fluorescent intensity than CC (Fig. 4b). Particularly for HUMC1, fluorescence spread out all over the dermis, even though it was brighter at the upper dermal layer. This is in accordance with the skin accumulation results that showed HUMCs favored skin disposition of hydrophilic molecules.

\section{DISCUSSION}

A HUMC vehicle composed of biocompatible ingredients was successfully developed for enhanced topical delivery of hydrophilic and lipophilic molecules. Carbopol hydrogel, which possesses excellent organoleptic characteristics and good patient acceptance, was selected as the polymeric base. Carbopol hydrogel shows high viscosity at low concentration. Moreover, this hydrogel retained water and decreased the loss of moisture from the skin surface following deposition of a film hindering water evaporation. ${ }^{15)}$ In order to form a gel structure, a neutralizing agent (e.g., triethanolamine) must be added to increase the $\mathrm{pH}$ and to thicken the carbopol dispersion. Due to the possible allergenicity of amine compounds, ${ }^{18)}$ carnosine was used as an alternative in this study. Carnosine 
is a basic dipeptide of amino acids beta-alanyl-L-histidine, which has antioxidant properties. ${ }^{19)}$

To amplify skin hydration effect, urea and Tinocare GL were added to the hydrogel vehicle as humectants. Urea, a natural moisturizing factor, is beneficial for atopic skin since it helps reduce trandsepidermal water loss. ${ }^{20)}$ Tinocare GL (1\% scleroglucan polysaccharide solution), a commercial product, reduces skin roughness and even shows anti-inflammatory activity. ${ }^{21)}$ Cetyl alcohol and grape seed oil were added to the hydrogel vehicle as emollients to impair water loss from SC. Cetyl alcohol is commonly used in cosmetic industry, since it makes SC oilier than other types of alcohols. Grape seed oil is rich in linoleic acid, which is predominantly found in epidermal phospholipids. Linoleic acid plays a critical role in barrier function because it is incorporated in ceramides, which are major components of intercellular lipids. ${ }^{22)}$ Olivem 1000 and DL- $\alpha$-tocopherol acetate were introduced to the hydrogel vehicle with humectants, as an emulsifier and an antioxidant, respectively. Olivem 1000 is obtained from olive oil by the esterification of its fatty acid groups with sorbitol and cetostearyl alcohol. It can be used as a non-irritant emulsifier by forming liquid crystals in moisturizing cream. With this composition, the HUMC vehicle established an $\mathrm{O} / \mathrm{W}$ system that provides non-greasiness with water-washable property, and had a $\mathrm{pH}$ value similar to $\mathrm{SC}(\mathrm{pH} 4.5-6) .^{23)}$

The hydrogel and HUMC vehicles showed similar patterns of plastic flow. The networked gel structures of the hydrogel and HUMC vehicles gave them strength to resist at low shear stress but flow when the shear stress becomes greater than the yield stress. Figure 1b shows a pseudoplastic flow imposed on Bingham flow, which possesses a yield point, i.e., it initially behaves like a Bingham material, but after yielding, it behaves like a power-law material. The simplest mathematical model for this type of material is known as the Herschel-Bulkley model and is given by the following equation: $T=T_{0}+k G$, where $T$ and $G$ are shear stress and shear rate and $T_{0}$ and $k$ are the yield stress and the viscosity, respectively. ${ }^{24)}$ If we compare the $T_{0}$ values $\left(\mathrm{dyn} / \mathrm{cm}^{2}\right)$ of different formulations, the ascending order was hydrogel $(373.74)<$ HUMC1 $(384.02)<$ HUMC2 (396.79)<HUMC3 (481.47). However, after a threshold, the slopes (viscosity) became similar, since the structured network collapsed and turned into a flowing liquid. This phenomenon is known as rheomalaxis, e.g., the permanent loss of viscosity of a high-molecular-weight polymer solution subjected to high shear rates. ${ }^{25)}$ High shear rates are capable of breaking covalent bonds in polymers, resulting in low molecular weight polymers with decreased solution viscosity.

We tested skin hydration effects of various formulations such as hydrogel, CC, HUMCs, and commercial products, and compared the degree of skin hydration in terms of $\Delta \mathrm{AU}$ by measuring the differences between untreated (before treatment) and treated (after treatment) groups of hairless mice. Hydrogel formed a gel structure and then moisturized the skin layer, but its hydration effect was relatively weak and rapidly disappeared. This is because hydrogel generally tends to dry quickly from solvent evaporation. ${ }^{16)} \mathrm{HUMC}$ and $\mathrm{CC}$ formulations exhibited better skin hydration capabilities. The addition of solid lipid and oil components would bring enhanced skin hydration by their occlusive properties. Among the formulations tested (including a few commercialized moisturizing creams), HUMC2 showed the best results in terms of skin hydration. The improved hydration effect of HUMC2 comes from the properties of both hydrogel and cream bases. It is a kind of synergistic effect between moisture absorption by the polymeric gel base and occlusion by solid lipid components: stearic acid and/or cetyl alcohol. In contrast, HUMC1 was less effective in skin hydration, because it did not contain solid lipid components. Solid lipids contributed to skin hydration by forming a film that prevented water evaporation from the skin surface. ${ }^{26)}$ Although HUMC3 contained a greater amount of solid lipids in the formulation, the hydration effects of HUMC3 were even less than those of HUMC1 and HUMC2. This might be attributed to the balanced ratio of oil and water in the formulation. HUMC2 is well organized with a networked gel structure in a balanced $\mathrm{O} / \mathrm{W}$ cream base. Subsequently, the hydration effect of HUMC2 was peaked within 30 min and continued for an hour, then maintained the highest level up to $360 \mathrm{~min}$ compared to other formulations.

In this experiment, to evaluate the drug delivery capacity of the selected formulations (HUMC1, HUMC2, and CC), NR and 5-CF were introduced as model probes for hydrophobic and hydrophilic molecules, respectively. As shown in Fig. 3 and Table 4, HUMCs revealed significantly enhanced permeation of both hydrophobic and hydrophilic molecules in comparison with CC. In particular, HUMC2, which contains adequate amounts of cetyl alcohol and stearic acid, resulted in the greatest effect on skin permeation. Previous studies have shown that a formula containing fatty acids and urea enhanced the skin permeation of the drug than control formula. ${ }^{27,28)}$ The increased permeation of NR and 5-CF by HUMC formulation may be associated with the permeation enhancing activity of those emollients and/or other constituents including solid lipids. Further investigation is necessary to elucidate the mechanism for enhanced skin delivery of HUMCs.

Skin penetration enhancement could be correlated with the skin hydration effects of the vehicle. Many corroborating studies have showed that hydration of the SC could alter permeant solubility, thereby modify partitioning from the vehicle into the membrane and thus increasing the penetration rate of drugs. These studies also found that increased skin hydration may result in swelling and opening of the compact structure of the SC, leading to an increase in penetration of drugs. ${ }^{13,29)}$ On the contrary, numerous papers concluded that skin hydration does not correlate with skin permeability. Although the use of solid nanoparticles increased skin hydration to a level similar to that achieved with the use of an ointment, the permeation rate of tacrolimus in a lipid nanoparticle formulation was almost 16-21 times higher than that of an ointment formulation. ${ }^{30)}$ The choice of ingredients is also a major factor influencing permeation enhancement. The flux values of two model drugs (diclofenac sodium and caffeine) from a propylene glycol-containing emulsion were higher than those from a glycerol-containing emulsion, although greater skin hydration was achieved with the glycerol-containing emulsion. ${ }^{31)}$

In the present study, the levels of skin hydration and permeation were varied based on the formulation type and the property of probe molecules. To investigate whether skin hydration correlates with skin permeation or accumulation of the drug molecule, Fig. 5 was created. For comparison, the extent of skin hydration was expressed as the area under the curve $\left(A U C_{\text {hydration }} ; \Delta \mathrm{AU} \cdot \mathrm{min}\right)$, calculated by the trapezoidal rule from $\Delta$ AU-time plots in Fig. 2A. The order of $A U C_{\text {hydration }}$ for 

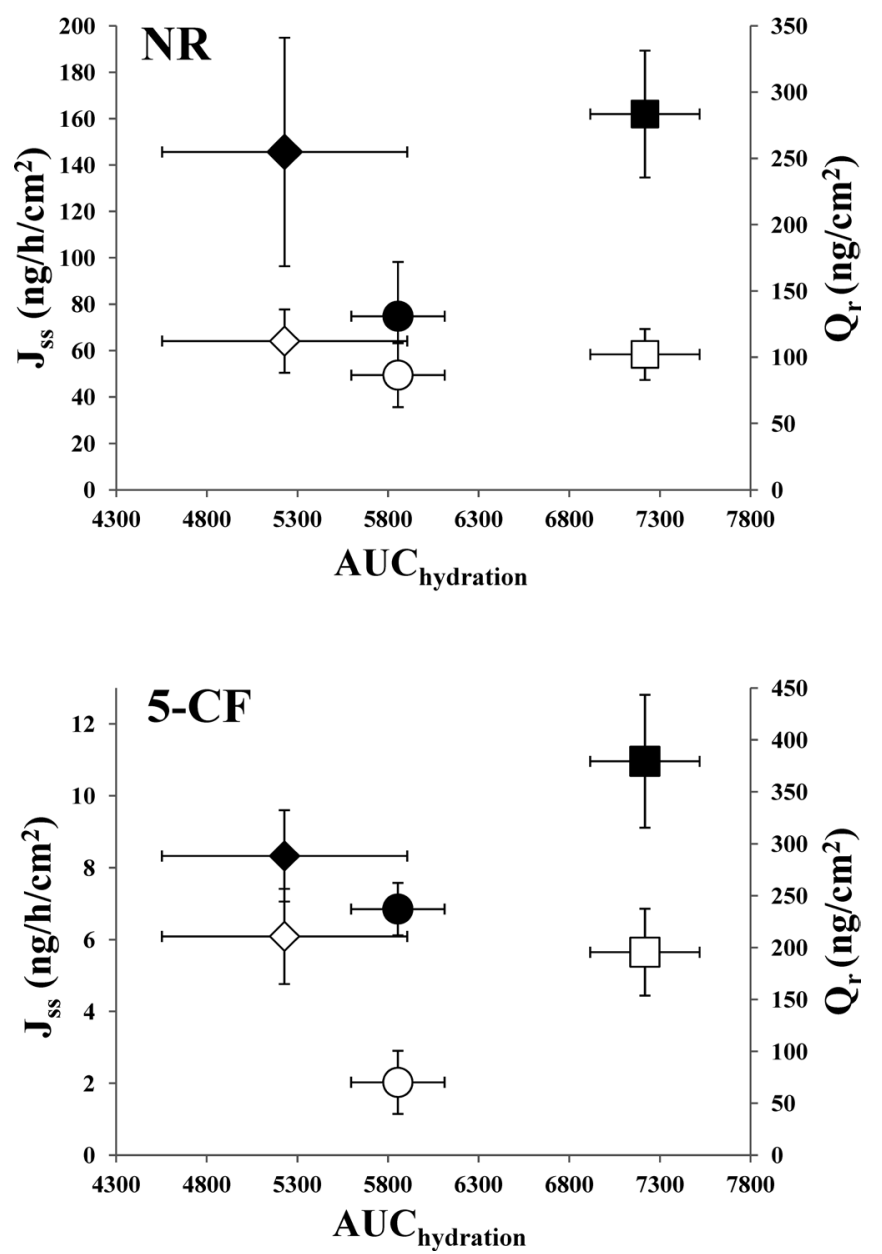

Fig. 5. Correlation of Skin Hydration ( $A U C_{\text {hydration }}$ : $x$-Axis) with the Steady-State Flux Value ( $J_{\text {ss }}$ : Left $y$-Axis, Filled Symbols) and the Amount Retained in the Skin at $12 \mathrm{~h}\left(Q_{\mathrm{r}}\right.$ : Right $y$-Axis, Open Symbols) for Three Formulations: Conventional Cream (CC, Circle); HydrogelBased Ultra-moisturizing Cream 1 (HUMC1, Diamond); and HUMC2 (Square)

Data represent mean \pm S.E.

selected formulation was as follows: HUMC2 (7216.9) $>\mathrm{CC}$ (5854.3) > HUMC1 (5229.0). The capacity for dermal delivery was compared by plotting the data points for permeation and the amount of drug in the skin at $12 \mathrm{~h}$. However, regardless of the physicochemical property of the molecules tested, the correlations were very poor in all instances. Although the permeation of both NR and 5-CF was increased by HUMC formulations, the increment was not proportional to $A U C_{\text {hydration }}$. A similar pattern was also observed for skin accumulation. Nevertheless, HUMC2 showed the superiority over other formulations in skin hydration and drug permeation. Therefore, we could conclude that enhanced dermal delivery with HUMC formulations was not only attributed to the skin hydration, but also to the balanced composition including adequate amounts of emollients and/or solid lipids.

In addition to the type of vehicles tested, the distribution and localization of drugs in the skin depend on the physicochemical properties of the drug molecules. As shown in Fig. 4 , due to the lipophilicity of NR as compared to 5-CF, NR localized in lipophilic parts of skin layer like adipose tissue before entering the receptor fluid. However, 5-CF was distributed homogeneously throughout the skin, and the relatively large amounts of 5-CF remained in SC layer. This result is consistent with an earlier report that hydrophilic dye from $\mathrm{O} / \mathrm{W}$ emulsion favored distribution onto the skin surface causing a homogeneous depth of penetration over the dermal area. ${ }^{32}$ The amount of 5-CF retained in the skin was significantly different between CC and HUMCs, but the amount of NR retained in the skin was similar in all formulations. Finally, we suggest that HUMC2 is an efficacious vehicle for skin hydration, and enhances dermal delivery of both lipophilic and hydrophilic drug molecules, particularly favoring the skin localization of hydrophilic molecules.

\section{CONCLUSION}

HUMC vehicles were successfully developed for skin hydration and enhanced dermal drug delivery, using biocompatible ingredients such as carbopol 934P as polymeric base, urea and Tinocare GL as humectants, grape seed oil as an emollient, and other excipients. HUMCs have desirable physical properties for external application, showing plastic flow behavior due to a networked gel structure in a balanced $\mathrm{O} / \mathrm{W}$ cream. In particular, the HUMC2 formulation containing adequate amounts of cetyl alcohol and stearic acid resulted in the greatest effect on both skin hydration and dermal permeation of either lipophilic or hydrophilic drug molecules. Therefore, the HUMC2 vehicle could be a promising tool for effective topical delivery of drug molecules, especially for the treatment of skin dryness and/or skin disorders such as atopic dermatitis (AD).

Acknowledgment This study was supported by a Grant from the Korea Healthcare Technology R\&D Project, Ministry for Health, Welfare and Family Affairs, Republic of Korea (A091121).

\section{REFERENCES}

1) Proksch E, Fölster-Holst R, Jensen JM. Skin barrier function, epidermal proliferation and differentiation in eczema. J. Dermatol. Sci., 43, 159-169 (2006).

2) Bouwstra JA, Ponec M. The skin barrier in healthy and diseased state. Biochim. Biophys. Acta, 1758, 2080-2095 (2006).

3) Akdis CA, Akdis M, Bieber T, Bindslev-Jensen C, Boguniewicz M, Eigenmann P, Hamid Q, Kapp A, Leung DY, Lipozencic J, Luger TA, Muraro A, Novak N, Platts-Mills TA, Rosenwasser L, Scheynius A, Simons FE, Spergel J, Turjanmaa K, Wahn U, Weidinger S, Werfel T, Zuberbier T; European Academy of Allergology; Clinical Immunology/American Academy of Allergy, Asthma and Immunology/PRACTALL Consensus Group. Diagnosis and treatment of atopic dermatitis in children and adults: European Academy of Allergology and Clinical Immunology/American Academy of Allergy, Asthma and Immunology/PRACTALL Consensus Report. Allergy, 61, 969-987 (2006).

4) Homey B, Steinhoff M, Ruzicka T, Leung DY. Cytokines and chemokines orchestrate atopic skin inflammation. J. Allergy Clin. Immunol., 118, 178-189 (2006).

5) Bikowski J. The use of therapeutic moisturizers in various dermatologic disorders. Cutis, 68 (Suppl.), 3-11 (2001).

6) Grimalt R, Mengeaud V, Cambazard F, Study Investigators' Group. The steroid-sparing effect of an emollient therapy in infants with atopic dermatitis: a randomized controlled study. Dermatology, 214, 61-67 (2007).

7) Chularojanamontri L, Tuchinda P, Kulthanan K, Pongparit K. 
Moisturizers for acne: What are their constituents? J. Clin. Aesthet. Dermatol., 7, 36-44 (2014).

8) Lodén M. The increase in skin hydration after application of emollients with different amounts of lipids. Acta Derm. Venereol., 72, 327-330 (1992)

9) Lodén M, Andersson AC, Andersson C, Frödin T, Oman H, Lindberg M. Instrumental and dermatologist evaluation of the effect of glycerine and urea on dry skin in atopic dermatitis. Skin Res. Technol., 7, 209-213 (2001)

10) Harding CR. The stratum corneum: structure and function in health and disease. Dermatol. Ther., 17 (Suppl. 1), 6-15 (2004).

11) Sinha VR, Kaur MP. Permeation enhancers for transdermal drug delivery. Drug Dev. Ind. Pharm., 26, 1131-1140 (2000).

12) Pathan IB, Setty CM. Chemical penetration enhancers for transdermal drug delivery systems. Trop. J. Pharm. Res., 8, 173-179 (2009).

13) Caussin J, Groenink HWW, de Graaff AM, Gooris GS, Wiechers JW, van Aelst AC, Bouwstra JA. Lipophilic and hydrophilic moisturizers show different actions on human skin as revealed by cryo scanning electron microscopy. Exp. Dermatol., 16, 891-898 (2007).

14) Bárány E, Lindberg M, Lodén M. Unexpected skin barrier influence from nonionic emulsifiers. Int. J. Pharm., 195, 189-195 (2000).

15) Tamburic S, Craig DQ. Rheological evaluation of polyacrylic acid hydrogels. Pharm. Pharmacol. Commun., 1, 107-109 (1995).

16) Scherer GW. Theory of drying. J. Am. Ceram. Soc., 73, 3-14 (1990).

17) $\mathrm{Ho} \mathrm{HO}$, Hsiao C, Sheu M. Preparation of microemulsions using polyglycerol fatty acid esters as surfactant for the delivery of protein drugs. J. Pharm. Sci., 85, 138-143 (1996).

18) Lessmann H, Uter W, Schnuch A, Geier J. Skin sensitizing properties of the ethanolamines mono-, di-, and triethanolamine. Data analysis of a multicentre surveillance network (IVDK) and review of the literature. Contact Dermatitis, 60, 243-255 (2009).

19) Babizhayev MA. Biological activities of the natural imidazole-containing peptidomimetics $n$-acetylcarnosine, carcinine and 1-carnosine in ophthalmic and skin care products. Life Sci., 78, 2343-2357 (2006).

20) Andersson A, Lindberg M, Loden $M$. The effect of two ureacontaining creams on dry, eczematous skin in atopic patients. I. Expert, patient and instrumental evaluation. J. Dermatol. Treat., 10, 165-169 (1999).
21) Coviello T, Palleschi A, Grassi M, Matricardi P, Bocchinfuso G, Alhaique F. Scleroglucan: a versatile polysaccharide for modified drug delivery. Molecules, 10, 6-33 (2005).

22) McCusker MM, Grant-Kels JM. Healing fats of the skin: the structural and immunologic roles of the omega- 6 and omega-3 fatty acids. Clin. Dermatol., 28, 440-451 (2010).

23) Schmid-Wendtner M-H, Korting HC. The $\mathrm{pH}$ of the skin surface and its impact on the barrier function. Skin Pharmacol. Physiol., 19, 296-302 (2006).

24) Huang $X$, Garcia MH. A Herschel-Bulkley model for mud flow down a slope. J. Fluid Mech., 374, 305-333 (1998).

25) Steffe JF. Mixer viscometry. Rheological Methods in Food Process Engineering (2nd ed.), Freeman Press, East Lansing, MI, pp. 185-210 (1992).

26) Choi WS, Cho HI, Lee HY, Lee SH, Choi YW. Enhanced occlusiveness of nanostructured lipid carrier (NLC)-based carbogel as a skin moisturizing vehicle. J. Pharm. Invest., 42, 243-250 (2012).

27) Kim CK, Kim J, Chi S, Shim C. Effect of fatty acids and urea on the penetration of ketoprofen through rat skin. Int. J. Pharm., 99, 109-118 (1993).

28) Lee JH, Chun IK. Effects of various vehicles and fatty acids on the skin permeation of lornoxicam. J. Pharm. Invest, 42, 235-241 (2012).

29) Van Hal DA, Jeremiasse E, Junginger HE, Spies F, Bouwstra JA. Structure of fully hydrated human stratum corneum: a freezefracture electron microscopy study. J. Invest. Dermatol., 106, 89-95 (1996).

30) Pople PV, Singh KK. Targeting tacrolimus to deeper layers of skin with improved safety for treatment of atopic dermatitis. Int. J. Pharm., 398, 165-178 (2010).

31) Savic S, Weber C, Tamburic S, Savic M, Müller-Goymann C. Topical vehicles based on natural surfactant/fatty alcohols mixed emulsifier: the influence of two polyols on the colloidal structure and in vitro/in vivo skin performance. J. Pharm. Sci., 98, 2073-2090 (2009)

32) Jacobi U, Tassopoulos T, Surber C, Lademann J. Cutaneous distribution and localization of dyes affected by vehicles all with different lipophilicity. Arch. Dermatol. Res., 297, 303-310 (2006). 